Mots. Les langages du politique

$94 \mid 2010$

Trente ans d'étude des langages du politique

(1980-2010)

\title{
Argumentation et discours politique
}

Ruth Amossy et Roselyne Koren

\section{(2) OpenEdition}

Journals

Édition électronique

URL : https://journals.openedition.org/mots/19843

DOI : $10.4000 /$ mots. 19843

ISSN : 1960-6001

Éditeur

ENS Éditions

\section{Édition imprimée}

Date de publication : 30 novembre 2010

Pagination : 13-21

ISBN : 978-2-84788-235-3

ISSN : 0243-6450

Référence électronique

Ruth Amossy et Roselyne Koren, "Argumentation et discours politique », Mots. Les langages du politique [En ligne], 94 | 2010, mis en ligne le 17 décembre 2012, consulté le 28 avril 2022. URL : http:// journals.openedition.org/mots/19843; DOI : https://doi.org/10.4000/mots.19843 


\section{Mots \\ Les langages du politique}

№ 94 novembre 2010

\section{Trente ans d'étude des langages du politique (1980-2010)}

ouvrage coordonné par Paul BACOT, Marlène Coulomb-GulLY, Jean-Paul Honoré, Christian Le BART, Claire Oger, Christian Plantin

\section{SOMMAIRE}

Paul Bacot, Marlène Coulomb-Gully, Jean-Paul Honoré, Christian Le Bart, Claire Oger, Christian Plantin

Le discours politique n'est pas transparent. Permanence

et transformations d'un objet de recherche

OUTILS ET ENJEUX DU DISCOURS POLITIQUE

Ruth Amossy, Roselyne Koren

Argumentation et discours politique

Christian Plantin

Argumentation-rhétorique. Les eaux mêlées

Caroline Ollivier-Yaniv

Discours politiques, propagande, communication, manipulation 
Marc Bonhomme

La caricature politique

Paul Bacot

Développement et diversification d'une onomastique politique

Ruth Wodak

The Discursive Construction of History. Brief Considerations

Henri Boyer

Les politiques linguistiques

\section{LIEUX DE LA PRODUCTION DU DISCOURS POLITIQUE}

Christian Le Bart

Parler en politique

Dominique Maingueneau

Le discours politique et son «environnement»

Alice Krieg-Planque, Claire Oger

Discours institutionnels. Perspectives pour les sciences

de la communication

Sophie Béroud, Josette Lefèvre

Le corpus syndical. Une expérience au long cours

Corinne Gobin, Jean-Claude Deroubaix

L'analyse du discours des organisations internationales.

Un vaste champ encore peu exploré

François de la Bretèque

Le retour de la parole politique dans le cinéma français

Jean-Claude Soulages

Vie et mort du citoyen cathodique

Jacques Guilhaumou

Les discours de la Révolution française.

Aperçu d'ensemble d'un trajet de recherche (1980-2009) 


\section{DISCIPLINES ET CHAMPS DE RECHERCHE POUR L'ÉTUDE DES LANGAGES DU POLITIQUE}

Philippe Braud

L'apport de la science politique à l'étude des langages du politique

Claire Blandin

L'apport de l'histoire des médias à l'étude des langages du politique

Jean-François Tétu, Bernard Lamizet

Les SIC et les langages du politique

Sylvianne Rémi-Giraud

Sémantique lexicale et langages du politique.

Le paradoxe d'un mariage difficile?

Marlène Coulomb-Gully, Juliette Rennes

Genre, politique et analyse du discours.

Une tradition épistémologique française gender blind

Johannes Angermüller

Analyser le discours politique en Allemagne (1980-2010)

Érik Neveu

L'apport de Pierre Bourdieu à l'analyse du discours.

D'un cadre théorique à des recherches empiriques

Roselyne Ringoot

Questionner le discours avec Michel Foucault.

Actualisations théoriques et actualité éditoriale

\section{ENTRETIEN}

Maurice Tournier

Mots et politique, avant et autour de 1980

La revue Mots. Les langages du politique encourage l'usage des rectifications de l'orthographe proposées par le Conseil supérieur de la langue française et approuvées par l’Académie (Journal officiel, $n^{\circ}$ 100, 6 décembre 1990). 


\section{Ruth Amossy \\ Roselyne Koren}

\section{Argumentation et discours politique}

Peut-on analyser le discours politique sans tenir compte de l'argumentation? Chez Aristote, le discours délibératif, destiné à réguler la vie de la Cité, est au centre du dispositif rhétorique. Fondé sur l'exhortation et la dissuasion, il vise l'avenir en termes d'avantages et d'inconvénients. C'est en des termes similaires qu'on définit aujourd'hui la communication politique qui, en régime démocratique, tente de faire adhérer les destinataires aux choix politiques qui leur sont proposés (Gerstlé, 2008, p. 79). Si l'on suit l'évolution des études sur le discours politique menées en France dans le domaine des sciences du langage et de la communication, on s'aperçoit pourtant que l'étude de l'argumentation est loin d'y tenir une place centrale.

Cet état de fait tient à diverses raisons, dont la première relève d'un état du champ : la linguistique accorde encore peu de place aux théories de l'argumentation rhétorique (par opposition à l'argumentation linguistique de Ducrot) ; elles n'ont pénétré que tardivement, et très partiellement, dans l'analyse du discours. Mais d'autres raisons tiennent à des positions de principe sur le discours politique. La première déplore sa décadence, topos dont l'ouvrage de Simone Bonnafous et al. (2003) a montré la persistance au cours des âges, en affirmant que le logos comme parole et raison s'efface de plus ou en plus au profit de l'ethos, de l'appel au sentiment et de la séduction. La seconde, plus radicale, consiste à se situer dans le sillage de Pierre Bourdieu pour dénoncer les pouvoirs de la parole et attribuer son autorité à la seule légitimité institutionnelle (Le Bart, 1998, p. 97-108). Malgré ces diverses résistances, les fructueux travaux réalisés durant ces trois dernières décennies reviennent néanmoins par des biais divers à la question de l'argumentation, ouvrant la voie à des entreprises d'investigation plus systématiques.

En un premier temps, il importe de préciser les sens dans lesquels on peut prendre la notion d'argumentation, comme d'ailleurs celle de discours politique : ces préliminaires sont indispensables pour déterminer ce qui peut être englobé dans la rubrique «argumentation et discours politique». Pour

ADARR, Tel-Aviv

amossy@attglobal.net

ADARR, Bar-Ilan

koren1@mail.biu.ac.il

Mots. Les langages du politique $n^{\circ} 94$ novembre $2010 \bullet 13$ 
Christian Le Bart, comme pour de nombreux autres chercheurs, le discours politique est celui que tiennent les hommes et femmes politiques dans l'exercice de leurs fonctions (1998, p. 7). Il s'agit là d'une définition ciblée et restreinte, à laquelle s'oppose une définition plus large et délibérément un peu floue, à savoir « tout propos qui implique un jugement sur l'organisation de la communauté» (Bonnafous et al., 2003, p. 12). L'objet d'étude va ainsi de la parole professionnelle des politiciens à tous les discours qui traitent de la chose publique dans l'espace public. C'est dans cette perspective que les divers travaux pris ici en compte explorent une pluralité de situations allant de l'orateur face à son auditoire, du débat parlementaire ou télévisé et du journalisme politique à la parole participative sur le Net.

Quant à l'argumentation, elle peut être définie comme un raisonnement cohérent qui mène à une conclusion par déduction ou induction, comme une logique des valeurs et du préférable inscrite dans les moyens verbaux destinés à faire adhérer l'auditoire à une thèse (Perelman, Olbrechts-Tyteca, 1958) ou comme un fonctionnement discursif qui oriente des façons de penser et de voir (Amossy, 2006). Dans le premier cas, on considère que seuls les arguments et les séquences argumentatives relevant d'une conception «théorique» formelle de la raison sont à prendre en ligne de compte. Dans le second cas, on considère qu'un jeu d'influences mutuelles s'exerce dans l'échange verbal qui recherche un accord sur le raisonnable; ce jeu d'influences se situe dans les espaces discursifs de la «raison pratique ». Dans la troisième éventualité, l'argumentation traverse le discours tout entier selon des modalités qui vont de la polémique à la co-construction des réponses. On retiendra une conception englobante qui tient compte de la raison pratique gérant l'évaluation, la décision et l'action politiques, inclut les modalités indirectes d'argumentation et accorde une importance égale au logos, au pathos et à l'ethos. On considérera par ailleurs que l'argumentation politique relève aussi bien de la recherche du consensus (but que lui assignent la plupart des théories de l'argumentation) que de la gestion du dissensus qui est au cœur de la vie politique, par définition conflictuelle. Dans cette perspective, on présentera brièvement un tableau raisonné (non chronologique) des acquis dans le domaine francophone.

Certains travaux se focalisent sur les arguments qui caractériseraient le discours politique. Bertrand Buffon (2002) propose ainsi un chapitre sur la « rhétorique politique» qui étudie, sur la base de la nouvelle rhétorique, les types d'arguments (liaison, dissociation, métaphore...) utilisés dans le champ politique. André Gosselin (1995) se penche sur les arguments de la cause en croisant la théorie des attributions causales et la rhétorique pour éclairer le «biais de complaisance» : les instances politiques expliquent leurs succès par des causes internes valorisantes, leurs échecs par des causes externes incontrôlables (et vice-versa pour les adversaires). Gilles Gauthier (1995), quant à lui, explore le décentrement des arguments ad rem vers les arguments ad homi- 
nem, soit l' «argumentation périphérique » ou ensemble des procédés discursifs qui se rapportent à la polémique, en répertoriant les divers types d'arguments ad hominem politiques - l'argument de la girouette, du tartuffe, de l'homme de paille, etc. Catherine Kerbrat-Orecchioni $(1981$, p. 45) fait un recensement de «quelques "fautes" argumentatives» constitutives de la mauvaise foi, relançant ainsi par un autre biais la question des paralogismes chère à la logique informelle.

Un autre type de travaux se focalise sur une rhétorique politique singulière. Albert O. Hirschman (1991) avait ouvert la voie dans son étude des trois arguments qui alimentent le discours réactionnaire depuis deux siècles en bloquant le débat: l'argument de l'effet pervers (ce qui doit permettre de progresser produit en fait le contraire), de l'inanité (le changement est impossible) et de la mise en péril (le changement met en danger des acquis). Il dégage ainsi les argumentaires qui fondent une «rhétorique réactionnaire» en offrant un modèle pour l'étude transversale d'autres rhétoriques politiques (libérale, progressiste, etc.). Marc Angenot met l'accent sur les topiques de discours étudiés sur un axe temporel long : celles, par exemple, de La propagande socialiste (1997), la plus grande entreprise rhétorique des temps modernes, ou de la Rhétorique de l'anti-socialisme (2004), parole de combat qui rejette les positions adverses en dehors du raisonnable dans un long «dialogue de sourds». PierreAndré Taguieff (1984) explore les ressorts du discours démagogique à travers «la rhétorique du national-populisme » exemplifiée par le chef de file du Front national, Jean-Marie Le Pen : il fonctionne par réduction des incertitudes, accumulation d'affirmations simples, empilement de pseudo-évidences, construction d'une façade logique et d'une image acceptable. Roselyne Koren (1996) retrace les modalités spécifiques des enjeux argumentatifs du journalisme politique. Dans une approche plus sociologique, Uli Windish (1995) se penche sur l'argumentation du citoyen « ordinaire » qui participe activement à la démocratie délibérative en Suisse ; il met ainsi en évidence une argumentation qui a sa rationalité propre et diffère des modèles abstraits en cours.

C'est aussi l'argumentation politique du citoyen ordinaire qui est en cause dans les travaux de plus en plus nombreux consacrés aux échanges dans le cyberespace. L'autorité des institutions politiques représentatives y est déstabilisée et contestée par les mises en cause critiques de la société civile. La Toile permet ainsi aux internautes, démontre Galia Yanoshevsky (2009a), de s'immiscer dans la gestion de l'ethos du candidat en lui faisant subir des modifications parfois incontrôlables; elle peut aussi donner à une notion perelmanienne aussi classique que celle d'auditoire particulier un éclairage inédit qui éclaire les enjeux de l'illusion de proximité (Yanoshevsky, 2009b). Les forums électroniques apparaissent comme un lieu alternatif de confrontation critique des points de vue qui instaure un nouveau type de démocratie participative. Certains y voient le déploiement de raisonnements qui ressemblent à des 
«monologues interactifs» en l'absence de véritable écoute et discussion. D'autres, comme Mathieu Chaput (2006 et 2008), montrent au contraire que le dispositiffavorise une grande interactivité, privilégiant des échanges courts et une "élaboration progressive de l'argumentation qui s'affine et se précise au contact des autres participants » (2006, p. 63). Dégager les spécificités de ces conversations numériques (éparpillement, fragmentation, recadrage permanent, construction d'un ethos discursif par des internautes masqués par un pseudonyme, etc.) s'avère alors indispensable pour analyser une discussion qui, hors de la portée des hommes de pouvoir et des journalistes politiques, se déroule au rythme de questions posées et gérées par les internautes dans un ordre informel et imprévisible. La rhétorique des échanges politiques sur le Net est considérée par les uns comme un tournant culturel bénéfique et par les autres comme une menace pour le fonctionnement de la démocratie représentative et la défense du bien commun.

D'autres travaux portent sur la rhétorique particulière de personnalités politiques. Dans les efforts pour contrer l'extrême droite, on s'est longuement penché sur l'argumentation de Jean-Marie Le Pen. Ainsi, Isabelle Cuminal et al. (1997) étudient une pratique discursive de l'évidence, du déplacement, de l'ambiguïté; Simone Bonnafous (2001) analyse l'arme de la dérision comme un mélange d'humour et d'agressivité dans le discours de Le Pen. Par ailleurs, Thierry Herman a publié en 2008 un ouvrage sur la rhétorique de Charles de Gaulle (1940-1945) et analysé l'éloge funèbre de François Mitterrand par Jacques Chirac (2001). De façon plus globale, on travaille également sur le fonctionnement argumentatif de certains genres relevant du champ politique : les débats politiques télédiffusés (Burger, 2005, Cabasino, 2007), les slogans (Amossy, 2006, dans la foulée de Reboul, 1975), les clips électoraux (Amossy, 2005). Raphaël Micheli $(2004,2008)$ explore le genre du débat parlementaire à partir d'un corpus portant sur l'abolition de la peine de mort. Dans ce cadre, il étudie aussi bien la question des stratégies de crédibilisation (relative à l'ethos), que l'argumentation qui vient justifier ou délégitimer une émotion (relative au pathos).

Dans ces différents contextes, l'accent est mis de façon de plus ou moins marquée sur la matérialité discursive. On trouve ainsi des analyses ponctuelles de procédés classiques comme la question rhétorique dans le discours parlementaire (Ducard, 2003), mais aussi de fonctionnements discursifs comme les enjeux argumentatifs de la nomination (Lecolle, 2007), des indications chiffrées (Koren, 2003), de l'exploitation des apparences de neutralité du récit (Adam, 1984, sur un discours de Giscard d'Estaing), de l'effacement énonciatif (Rabatel, 2006, sur un journal de campagne électorale), etc. Certains travaux, sans se consacrer exclusivement au discours politique, offrent des cadres d'analyse globaux qui permettent d'en éclairer concrètement les modalités argumentatives. Ainsi Jean-Michel Adam (1999) jette les fondements d'une linguistique 
textuelle au sein de laquelle il analyse la « rhétorique de l'appel : de Gaulle et Pétain en juin 1940 » en confrontant sur des bases langagières les dominantes argumentatives des deux discours. Après avoir proposé un cadre «au carrefour des disciplines » pour l'analyse de la construction de l'ethos, exemplifiée par un discours de Jean-Marie Le Pen et un écrit politique de Jean Giono (1999), Ruth Amossy développe une théorie de l'argumentation dans le discours (2010) qui situe l'argumentation au cœur de l'échange verbal et permet de traiter aussi bien des allocutions de Jean Jaurès à la Chambre que des manifestes d'intellectuels ou des interviews d'hommes politiques. Enfin, parmi les tentatives de cibler le discours politique comme tel, il faut signaler l'ouvrage de Patrick Charaudeau (2005a), Le discours politique. S'inscrivant dans une logique d'influence sociale, il minimise l'argumentation comme échange raisonné pour lui substituer la persuasion entendue comme séduction, voire manipulation. Selon lui, il s'agit tout au plus pour l'homme politique de «proposer un raisonnement causal simple en s'appuyant sur des croyances fortes [...] et de les renforcer en apportant des arguments destinés à produire un effet de preuve » (2005a, p. 77). C'est que le politique (lieu de fabrication et de confrontation heuristique des idées) le cède de plus en plus à la politique - «lieu d'exercice du pouvoir et d'influence pour faire partager les idées de la gouvernance » (ibid., p. 236). Dès lors, la mise en scène prime sur le contenu des idées et l'ethos l'emporte sur le logos.

Ces divers angles d'attaque confirment qu'il n'existe pas de conception intégrative consensuelle de l'argumentation ni, a fortiori, de l'argumentation politique. Ce qui soude toutefois le métadiscours critique des diverses approches, c'est - comme le souligne Pierre-André Taguieff (1990, p. 262-263) - «un ensemble de questions que se posent tous ceux qui s'interrogent sur les modes de fonctionnement, de production et d'efficacité du discours dans le champ du politique ». La lecture des travaux recensés permet de percevoir la place fondamentale occupée par diverses problématiques : 1 ) le refus de limiter la situation de communication politique type à celle de l'orateur face à son auditoire (Taguieff, 1990, p. 278; Windisch, 1995, p. 80). La recherche en argumentation politique aurait à se mesurer par exemple aujourd'hui aux formes inédites que revêt la politique participative sur la Toile (Barbet, Bonhomme, Rinn éd., 2006; Hermès, nº 47; Doury, Lefébure, 2006; Marcoccia, 2003); 2) la distinction et les rapports entre conviction et persuasion, où « convaincre » est associé à « raisonner» et donc au logos tandis que "persuader» est le plus souvent associé à «séduire» ou même «manipuler» et donc aux passions du locuteur décidé à imposer son point de vue de gré ou de force (Danblon, 2005; Charaudeau, 2005a, p. 62, 2005b, 2008). Cette distinction est liée par des liens essentiels à la problématisation des notions de rationalité et/ou d'irrationalité. Il s'agit tout particulièrement aujourd'hui de défendre, à l'encontre d'une conception cartésienne dogmatique du rationalisme, la thèse de la pluralité des régimes 
de rationalité possibles jugés aussi valides et légitimes les uns que les autres. Quelques théoriciens envisagent même la possibilité d'une éthique de la responsabilité, comparable à celle défendue par Weber, qui autoriserait l'homme politique à argumenter ses décisions à rebours des prescriptions de la raison dans le cas de conflits ou de tragédies hors normes (Taguieff, 1990; Gosselin, $1995)$; 3) la revalorisation du dissensus qui n'est plus présenté systématiquement comme un échec, mais comme un des moteurs démocratiques du politique et de ce fait comme un constituant intrinsèque du conflit, en tous points préférable aux violences de la guerre (Wolton, 1995, p.13; Amossy, 2010); 4) un questionnement éthique (Gauthier, 1995, p. 179; Koren, 2006 et 2008) qui accorde une place centrale aux controverses autour des notions de manipulation, et donc en creux de rectitude (Taguieff, 1990, p. 274; David-Blais, 1995; Breton, 1997; Danblon, 2005) ou de «bon» vs «mauvais» argument (Kerbrat-Orecchioni, 1981; Charaudeau, 2005b, p. 34-35) et donc à la question de la validité des normes argumentatives. Cette problématique repose la délicate question de la position du chercheur : l'analyse de l'argumentation politique doit-elle être purement descriptive ou au contraire également normative, lui faut-il rester neutre ou doit-elle être guidée par des principes éthiques qui débouchent sur la critique, voire parfois sur la dénonciation? Si la question concerne l'analyse du discours en général, elle se pose avec une particulière acuité dans le double champ de l'argumentation et de la politique. Il apparaît qu'elle est loin d'être résolue.

\section{Références}

ADAM J.-M., 1984. «Pour une pragmatique textuelle : l'exemple d'un discours politique giscardien », Le discours politique, C. Kerbrat-Orecchioni, M. Mouillaud éd., Lyon, Presses universitaires de Lyon, p.187-212.

- 1999, Linguistique textuelle. Des genres de discours aux textes, Paris, Nathan.

Amossy R., 1999, "L'ethos au carrefour des disciplines. Rhétorique, pragmatique, sociologie des champs ", Images de soi dans le discours. La construction de l'ethos, Lausanne, Delachaux \& Niestlé, p. 125-154.

- 2005, «Rhétorique et communication politique. L'exemple du clip électoral, Israël, 2003 ", Argumentation et communication dans les médias, M. Burger, G. Martel éd., Montréal, Nota Bene, p. 217-238.

- 2006 [2000], L'argumentation dans le discours, Paris, Armand Colin.

- 2010, "The functions of polemical discourse in the public sphere», The Responsabilities of Rhetoric, M. Smith, B. Warnick éd., Long Grove, Waveland Press, p. 52-61.

ANGENOt M., 1997, La propagande socialiste. Six essais d'analyse du discours, Montréal, Balzac.

- 2003, "Anarchistes et socialistes. Trente-cinq ans de dialogue de sourds», La parole polémique, G. Declercq, M. Murat, J. Dangel éd., Paris, Honoré Champion, p. 409-513. 
- 2004, Rhétorique de l'anti-socialisme. Essai d'histoire discursive 1830-1917, Québec, Presses de l’Université de Laval.

Barbet D., Bonhomme M., Rinn M. éd., 2006, Mots. Les langages du politique, no 80, mars, La politique mise au Net.

Bonnafous S., 2001, «L'arme de la dérision chez Jean-Marie Le Pen », Hermès, n²9, p. 53-63.

Bonnafous S., Ciron P., Ducard D., Levy C. éd., 2003, Argumentation et discours politique, Rennes, Presses universitaires de Rennes.

BRETON P., 1997, La parole manipulée, Paris, La Découverte.

BufFON B., 2002. La parole persuasive. Théorie et pratique de l'argumentation rhétorique, Paris, PUF.

BURGER M., 2005, "La complexité argumentative d'une séquence de débat politique médiatique », Argumentation et communication dans les médias, M. Burger, G. Martel éd., Montréal, Nota Bene, p. 51-79.

CABASINO F., 2007, «La construction de l'ethos présidentiel dans le débat télévisé français ", Mots. Les langages du politique, n 89, mars, p. 11-23.

CHAPUT M., 2006, «La dynamique argumentative des discussions politiques sur Internet», COMMposite, $\mathrm{n}^{\circ}$ 1, p. 52-77.

- 2008, «Analyser la discussion politique en ligne. De l’idéal délibératif à la reconstruction des pratiques argumentatives », Réseaux, n¹50, p. 83-106.

Charaudeau P., 2005a, Le discours politique. Les masques du pouvoir, Paris, Vuibert.

- 2005b, «Quand l'argumentation n'est que visée persuasive. L'exemple du discours politique », Argumentation et communication dans les médias, M. Burger, G. Martel éd., Montréal, Nota Bene, p. 29-49.

- 2008, "L'argumentation dans une problématique d'influence», Analyse du discours et argumentation, $\mathrm{n}^{\circ} 1$, en ligne [URL : http://www.tau.ac.il/ adarr/index. files/revue.html]. Consulté le 14 juin 2010.

Cuminal I., Souchard M., Wahnich S., Wathier V., 1997, Le Pen. Les mots. Analyse d'un discours d'extrême droite, Paris, Le Monde Éditions.

DAnblon E., 2005, La fonction persuasive, Paris, Armand Colin.

DAVID-BLAIS M., 1995, «L'argumentation nationaliste est-elle nécessairement irrationnelle? Le cas de la Pologne postcommuniste », Hermès, nº 16, p. 201-214.

Doury M., LefÉbure P., 2006, « “Intérêt général”, “Intérêts particuliers”. La construction de l'ethos dans un débat public », Questions de communication, nº 9, p. 47-71.

DuCARD D., 2003, «Une discussion biaisée. La question rhétorique dans le débat parlementaire », Argumentation et discours politique, S. Bonnafous et al. éd., Rennes, Presses universitaires de Rennes, p. 191-200.

GAUTHIER G., 1995, «L'argumentation périphérique dans la communication politique. Le cas de l'argument “ad hominem" ", Hermès, nº 16, Argumentation et rhétorique, p. 167-186.

GERSTLÉ J., 2008 [2004], La communication politique, Paris, Armand Colin.

Gosselin A., 1995, «Les attributions causales dans la rhétorique politique», Hermès, $\mathrm{n}^{\circ}$ 16, Argumentation et rhétorique, p. 153-166.

Herman T., 2001, «Le Président est mort, vive le Président». Images de soi dans l'éloge 
funèbre de François Mitterrand par Jacques Chirac », La mise en scène des valeurs, M. Dominicy, M. Frédéric éd., Lausanne, Delachaux \& Niestlé, p.167-202.

- 2008, Au fil des discours. La rhétorique de Charles de Gaulle, 1940-1945, Paris, Lambert-Lucas.

Hermès, 2007, n 47, Paroles publiques. Communiquer dans la cité, I, «Les modes de circulation des paroles publiques », F. Massit-Folléa, C. Méadel éd.

HIRSCHMAN A., 1991, Deux siècles de rhétorique réactionnaire, Paris, Fayard.

KERBRAT-ORECCHIONI C., 1981, "Argumentation et mauvaise foi », L'argumentation, Lyon, Presses universitaires de Lyon, p. 41-63.

Kerbrat-Orecchioni C., Mouillaud M., 1984, Le discours politique, Lyon, Presses universitaires de Lyon.

KOREN R., 1996, Les enjeux éthiques de l'écriture de presse et la mise en mots du terrorisme, Paris, L'Harmattan.

- 2003, "Contribution à l'étude des enjeux de la rhétorique laconique. Le cas des indications chiffrées», Topique, n83, Presse, sciences humaines et terrorisme, p. 111-124.

- 2006, "La responsabilité des uns dans le regard des autres. L'effacement énonciatif au prisme de la prise de position argumentative », Semen, n²2, p. 93-108.

— 2008, "Éhique de conviction” et/ou “éthique de responsabilité". Tenants et aboutissants du concept de responsabilité collective dans le discours de trois quotidiens nationaux français", Questions de communication, nº13, A. Rabatel, R. Koren éd., p. 25-45.

LE BART C., 1998, Le discours politique, Paris, PUF (Que sais-je ?).

LECOLLE M., 2007, «Enjeux argumentatifs de la nomination. Le cas du nom collectif communauté dans les discours publics contemporains», Argumentation, manipulation, persuasion, C. Boix éd., Paris, L'Harmattan, p. 227-248.

MARCoccia M., 2003, "Parler politique dans un forum de discussion», Langage et société, nº104, p. 9-55.

MICHELI R., 2004, «Justifier ou illégitimer la peine de mort? Aspects argumentatifs du débat parlementaire de $1981 »$, Mots. Les langages du politique, nº 74, mars, p.109-121.

- 2007, "Stratégies de crédibilisation de soi dans le discours parlementaire», A contrario, vol. $5, \mathrm{n}^{\circ} 1$, p. 67-84.

- 2008, «L'analyse argumentative en diachronie. Le pathos dans les débats parlementaires sur l'abolition de la peine de mort», Argumentation et analyse du discours, no 1, en ligne [URL: http://aad.revues.org/index482.html]. Consulté le 14 juin 2010.

Perelman C., Olbrechts-TyteCA L., 1958, Traité de l'argumentation. La nouvelle rhétorique, Bruxelles, Université de Bruxelles.

RABATEl A., 2006, "L'effacement de la figure de l'auteur dans la construction événementielle d'un “journal” de campagne électorale», Semen, n²2, p. 77-92.

Reboul O., 1975, Le slogan, Paris, PUF.

TAguieff P.-A., 1984, "La rhétorique du national-populisme», Mots. Les langages du politique, $\mathrm{n}^{\circ}$ 9, octobre, p. 113-139.

- 1990, "L'argumentation politique. Analyse du discours et nouvelle rhétorique», Hermès, nº 8-9, p. 261-283. 
WINDISCH U., 1995, «L'argumentation politique. Un phénomène social total», Année sociologique, vol. 45, $\mathrm{n}^{\circ} 2$, p. 59-82.

WINDISCH U., AMEY P., GRÉTILLAT F., 1995, «Communication et argumentation politiques quotidiennes en démocratie directe », Hermès, nº 16, p. 57-72.

Wolton D., 1995, «Avant-propos. Argumentation : le déficit d’analyse », Hermès nº 15 , p. 11-17.

YAnoshevsky G., 2009a, "L'usage des vidéoblogs dans l'élection présidentielle de 2007. Vers une image plurigérée des candidats ", Mots. Les langages du politique, n०89, mars, p. 57-68.

- 2009b, «Perelman's audience revisited : towards the construction of a new type of audience », Argumentation, n² 23, p. 409-419. 